\title{
Effect of fiber and solenoid variation parameters on the elements of a corrector PID for electromagnetic fiber squeezer based polarization controller
}

\author{
Abdallah Zahidi ${ }^{1}$, Said Amrane ${ }^{2}$, Nawfel Azami ${ }^{3}$, Naoual Nasser ${ }^{4}$ \\ 1,2,3 INPT Optics Lab,National Institute of Posts and Telecommunications, Rabbat, Morocco \\ ${ }^{4}$ LDEDS, Faculties of Science and Technology, Hassan University $1^{\text {st }}$, Settat, Morocco
}

\begin{tabular}{l} 
Article Info \\
\hline Article history: \\
Received Apr 21, 2019 \\
Revised Nov 13, 2019 \\
Accepted Nov 26, 2019 \\
\hline
\end{tabular}

Keywords:

Electromagnetic fiber squeezer

PID corrector

Polarization controller

Stability

\begin{abstract}
Controlling the polarization of the light output from single-mode fiber systems is very important for connecting it to polarization-dependent integrated optical circuits, while applications using a heterodyne detection system. Polarization controller using fiber squeezer is attractive for a lowloss, low-penalty coherent optical fiber trunk system. However, for polarization controllers using electromagnetic fiber squeezer, the stability problem due to the saturation of their magnetic circuit must be studied. In fact, in their conventional configuration, open-loop stability affects performance and limits applications. First at all, this effect has been analyzed and a feedback circuit with correctors has been proposed to improve stability performance. Then a simulation study is proposed to examine the influence of the system parameters on the corrector constants. The results of the simulation show that if the system parameters change the constants $\mathrm{Kp}$, $\mathrm{Ki}$ and $\mathrm{Kd}$ of the PID corrector must be adjusted to keep an optimized dynamic response.
\end{abstract}

Copyright $@ 2020$ Institute of Advanced Engineering and Science. All rights reserved.

\section{Corresponding Author:}

Abdallah Zahidi,

National Institute of Posts and Telecommunications,

Avenue Allal Al Fassi, Rabat, Morocco.

Email: zahidiabdo@yahoo.fr

\section{INTRODUCTION}

Many of the proposed applications of single-mode optical fibers employ polarized light. For example, a variety of fiber-optical sensors measuring absolute rotation, strain, temperature, sound, or current, are based on the interference of two waves that have propagated through two lengths of fiber. For correct operation the two waves must emerge in a suitable state of polarization (SOP). Similarly, integrated optical components for single-mode communications, such as switched directional couplers, perform properly only with one particular SOP at their input [1]. Currently available single-mode fibers, on the other hand, propagate in fact two modes of orthogonal polarizations with slightly different velocities, and moreover these two SOPs are coupled at even weak bends and twists. Consequently, while the fiber perfectly do not affect the degree of polarization of monochromatic light, the SOP is not maintained. A given SOP launched into the fiber evolves into other states after a short distance. Temperature variations and mechanical disturbances further exacerbate the problem of achieving a desired SOP at the fiber output in the abovementioned applications. To solve this problem, several techniques have been proposed, such as polarization control [2, 3], polarization diversity [4], polarization scrambling [5], and polarization switching [6]. Several technologies to realize polarization controller were introduced in the last two decades such as: mechanically rotating crystal wave plates or fiber loops, fiber squeezers or quartz with piezo electric elements[7], rotating wave plates [8, 9], variable retarder plates [10], liquid crystals [11], and waveguide devices such as LiNbO3 $[9,12]$. A polarization controller using fiber squeezers is promising because of its 
low insertion loss and low power penalty [13] which are particularly necessary for a main line system and used for static polarization matching between the polarization-sensitive components. In addition, the solenoids used by these controllers as fiber press are widely used in many home and industrial equipment in which an all-or-nothing mechanical movement is required.They are simple, robust and relatively inexpensive construction [14]. However for polarization controllers using these solenoids as a mechanical actuator, the problem of stability must be studied. Indeed, these devices have mechanically moving parts that limit their precise control and stability [15] and one potential drawback to this type of actuator is its limited stable travel range [16]. These problems are due to the saturation of the magnetic circuit of these controllers, which limits the phase shift of their operation and thus degrades the stability [17].

In a first step, the objective of this study is to investigate PID monitoring to control dynamic response of the output light intensity. In second step, we will analyze the effect of fiber and solenoid parameters variation on the PID corrector coefficients. This method use simulation electromagnetic fiber squeezer based polarization controller with function tfest of Malab to determinate transfer function system and pidtune for $\mathrm{PID}$ controller constants $\mathrm{Kp}, \mathrm{Kd}$ and $\mathrm{Ki}$ which allow obtaining a dynamic response output light intensity of the system with sufficient stability.

\section{MOTIVATION}

Solenoids also called electromagnetic actuator driven by nonlinear magnetic forces are widely used in many applications, such as positioners [18], valves [19-20], anti-braking systems [21], robotic manipulators [22] and polarization controllers where solenoids are used as mechanical actuator on the fiber to adjust the output light power. A potential disadvantage of this type of actuator lies in its stable load transport range, which accounts for less than a third of the full range [16]. Numerous techniques have been studied to extend the stable range of motion of these types of actuators, including nonlinear magnetic field mapping [23], double solenoid configuration [24], pulse width modulation control [25], sliding mode control [26], adaptive control [27], and the PID Control that are easy to design and implement [14]. However, the difficult task is to automatically and accurately adjust PID gains in case of solenoid variation parameters, which affect the performance of the solenoid-based polarization controller. This parametric variation may be due to changes in the wavelength value, the spring constant, the coefficient of friction, variations in the inductance and the resistance of the coil, etc.

This document is organized as follows. Section 2 describes the operating principle of electromagnetic fiber squeezer based polarization controller. Section 3 presents the mathematical modeling of the solenoid and electromagnetic fiber squeezer based polarization controller. Section 4 describes and analyzes closed-loop polarization controller simulation Matlab/Simulink with PID corrector as the influence of system parametric variables on correction constants for the design of an auto-tuning PID controller.

\section{STRUCTURE AND FUNCTIONALITY}

The birefringence of an optical fiber can be changed by squeezing it in radial direction [28]. Figure 1 shows the schematic diagram of a fiber squeezer. The device induce elastoopically adjustable amount of birefringence in the fiber. For that, a current dependent transversal force is induced on the fiber by placing a short length between frame and armature of solenoid Figure 1.

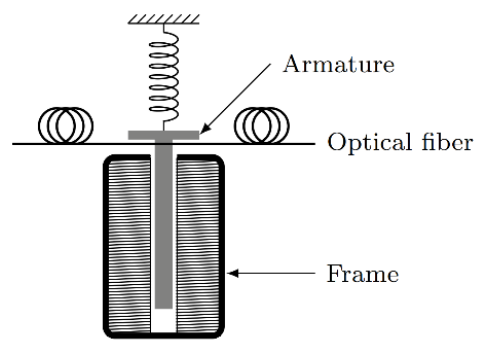

Figure 1. Optical fiber placed between the frame and solenoid armature

The magnitude of the phase difference of two polarized light along the squeezing axis and its orthogonal axis can be expressed as [29]:

$$
\delta=6.10^{-5} \frac{\mathrm{F}}{\lambda \mathrm{d}}
$$


and the light power $\mathrm{P}_{\mathrm{s}}$ at the output of the polarization analyzer according to scheme of Figure 2.

$$
P_{s}=E^{2}
$$

where

$$
\begin{aligned}
& \mathrm{E}=\mathrm{A} \cdot \frac{1}{2} \cdot \mathrm{E}_{0} \\
& \mathrm{~A}=\left(\begin{array}{cc}
\mathrm{e}^{\mathrm{j}\left(\phi_{\mathrm{m}}+\frac{\delta}{2}\right)} & 0 \\
0 & \mathrm{e}^{\mathrm{j}\left(\phi_{\mathrm{m}}-\frac{\delta}{2}\right)}
\end{array}\right) \\
& \mathrm{P}_{\mathrm{S}}=\mathrm{E}^{2}=\frac{\mathrm{P}_{0}}{2}(1+\cos \delta)
\end{aligned}
$$

where $\mathrm{P}_{0}$ is the input light intensity.

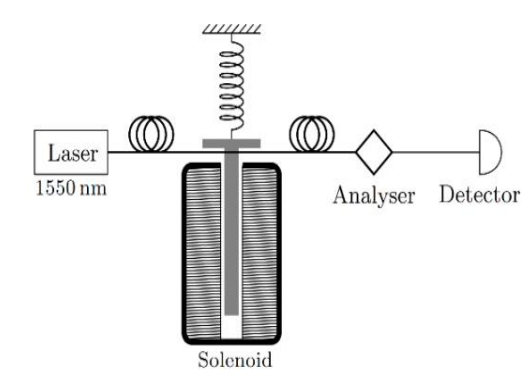

Figure 2. Scheme of using the electromagnetic squeezer fiber

\section{MATHEMATICAL MODEL}

\subsection{Mathematical model of solenoid}

The solenoid is the electromagnetic actuator. It is used to exert pressure on the fiber. It structure is shown in Figure 3.

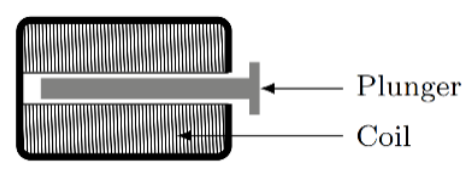

Figure 3. Cross section of solenoid

The mathematical model of solenoid is given [16] by the following expression :

$$
m \frac{d}{d t}\left(\frac{d x}{d t}\right)+\frac{c d x}{d t}+k x(t)=\frac{\mu_{0} \mu_{r} N^{2} A I^{2}(t)}{2\left(x_{0}-x(t)\right)^{2}}
$$

where:

$\mathrm{x}(\mathrm{t})$ : Displacement of the armature

$\mathrm{i}(\mathrm{t})$ : The electromagnet coil current (A)

A: the cross sectional area of the coil

$\mathrm{N}$ : the number of the turns of the coil

$\mu_{0}$ : Permeability of the free space

$\mu_{\mathrm{r}}$ : Relative Permeability of the dielectric materiel between the coil and armature

$\mathrm{x}_{0}$ : The initial air gap between the armature and the backside of the frame.

$\mathrm{m}$ : Masse of the armature

$\mathrm{k}$ : is the stiffness of the spring

c: System damping coefficient

Effect of fiber and solenoid variation parameters on the elements of a corrector PID ... (Abdallah Zahidi) 
The equation of the electrical circuit is as follows :

$$
\begin{aligned}
& u=R i(t)+\frac{d}{d t}[L(x) \cdot i] \\
& u=R i(t)+L(x) \frac{d i(t)}{d t}+i(t) \frac{d L(x)}{d t}
\end{aligned}
$$

$\mathrm{R} \quad$ : is the series resistance of the solenoid coil

$\mathrm{L}(\mathrm{x})$ : is the inductance of the coil that depends of the air gap [16]

$$
\mathrm{L}(\mathrm{x})=\frac{\mu_{0} \mu_{\mathrm{r}} A}{\mathrm{x}_{0}-\mathrm{x}(\mathrm{t})}
$$

\subsection{Mathematical model of polarization controller using the solenoid}

The balance equation of the force acting on the fiber is expressed as follow [30]:

$$
M \frac{d^{2} x}{d t^{2}}=F-k_{s}\left(x-x_{0}\right)-B \frac{d x}{d t}
$$

$\mathrm{k}_{\mathrm{S}}:$ is the spring constant.

$\mathrm{x}:$ is the displacement of the plunger.

$\mathrm{x}_{0}$ : is the equilibrium position of the plunger.

$B:$ is the coefficient of friction.

$\mathrm{F}:$ is the force produced by the magnetic field and it can be derived supposing that magnetic system is linear and that current is kept constant:

$$
\mathrm{F}=\frac{\mathrm{dw} t}{\mathrm{dx}}=\frac{\mathrm{i}^{2}}{2} \frac{\mathrm{dL}(\mathrm{x})}{\mathrm{dx}}=\frac{\mathrm{i}^{2}}{2} \frac{a L^{\prime}}{(\mathrm{a}+\mathrm{x})^{2}}
$$

where

$$
\mathrm{L}^{\prime}=\frac{\mu_{0} \cdot \pi \cdot a \cdot d \cdot N^{2}}{\mathrm{~g}}
$$

and a, d, g parameters depending on the solenoid geometry

The result modeling are the equations with input voltage $\mathrm{u}(\mathrm{t})$ and output light intensity $P_{S}$ from (8) we can write,

$$
\frac{d i}{d t}=\frac{1}{L(x)}\left(u-R i-i \frac{d L(x)}{d x} \frac{d x}{d t}\right)
$$

from (10) we can write

$$
\frac{\mathrm{d}^{2} \mathrm{x}}{\mathrm{dt}^{2}}=\frac{1}{\mathrm{M}}\left(\mathrm{F}-\mathrm{k}_{\mathrm{s}}\left(\mathrm{x}-\mathrm{x}_{0}\right)-\mathrm{B} \frac{\mathrm{dx}}{\mathrm{dt}}\right)
$$

both (1) and (5) are used to write:

$$
P_{S}=\frac{P_{0}}{2}\left(1+\cos \left(6.10^{-5} \frac{F}{\lambda d}\right)\right)
$$

\section{SIMULINK MODEL}

The system model has been implemented in versatile software MATLAB wish is widely used in control engineering around the word. This simulation is used to effectively determine the best performance dynamic response of the output light intensity. The electrical model Simulink which models (13) is represented in the Figure 4 while the Simulink mechanical model for (1) is illustrated in the Figure 5. The Figure 6 represents the optical Simulink model for (14). 


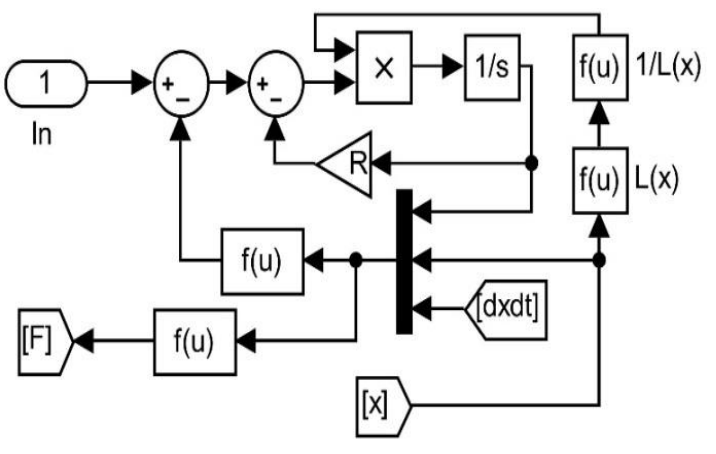

Figure 4. Electrical simulink model

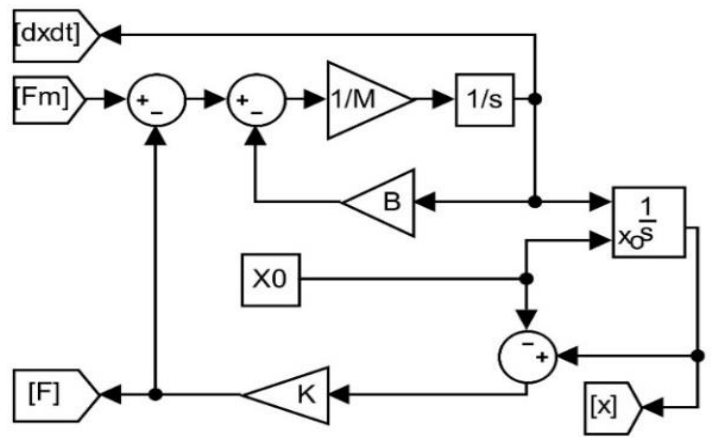

Figure 5. Mechanical simulink model

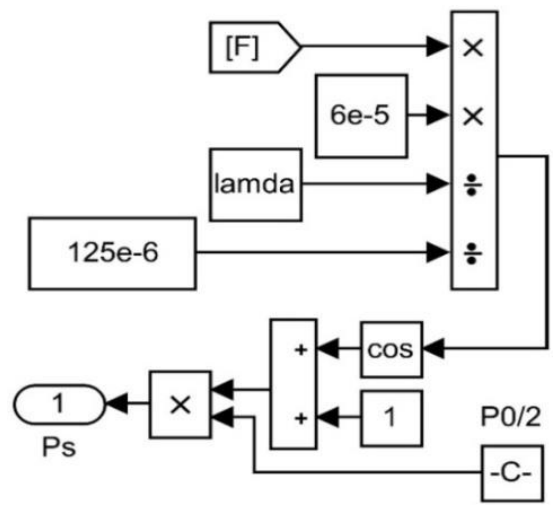

Figure 6. Optical simulink model

\section{SIMULATION AND RESULT}

\subsection{Principle of simulation}

First, we propose a mathematical model to obtain the response of the system, and then this model is identified from this response, looped and corrected using a PID controller feedback. Finally, a simulation-based study is used to examine the effect of the system parameters on the PID variables $(\mathrm{Kp}, \mathrm{Ki}$ and $\mathrm{Kd})$; parameters include those of fiber $(\lambda)$ and solenoid: mass of armature $(\mathrm{m})$, coefficient of friction $B$, spring stiffness $(K)$, coil resistance $(R)$ and inductance $(L)$. The ranges of values used for these parameters are as follows: $\lambda: 1100$ to $2000 \mathrm{~nm}, \mathrm{~m}: 100$ to $500 \mathrm{~g}, \mathrm{~B}: 2$ to $4 \mathrm{Ns} / \mathrm{m}, \mathrm{k}: 1000$ to $3000 \mathrm{~N} / \mathrm{m}$, $\mathrm{R}: 1$ to $50 \Omega$, L: 10 to $35 \mathrm{mH}$.

\subsection{Simulation flowchart} in Figure 7.

All the models are simulated using the MATLAB/ SIMULINK program whose flowchart is given

\subsection{Used function}

\subsubsection{Identification by tfest function}

The tftest function whose syntax is sys=tfest(data,np,nz) is used to estimate a transfer function containing nz zeros, np poles. For time domain estimation, data is an iddata object containing the input and output signal values. The model is estimated from an input data and output data time series that allow building a simulation model. The transfer function of this system can be estimated using the same input and output data using the tfest function for which an iddata object for the time series data is needed.

\subsubsection{Correction by pidtune function}

Pidtune tunes the parameters of the PID controller to balance performance (response time) and robustness (stability margins). Its syntax is pidtune (sys, PID, opts). Sys is a tunable or uncertain model, opts is additional tuning options such as the target phase margin. 


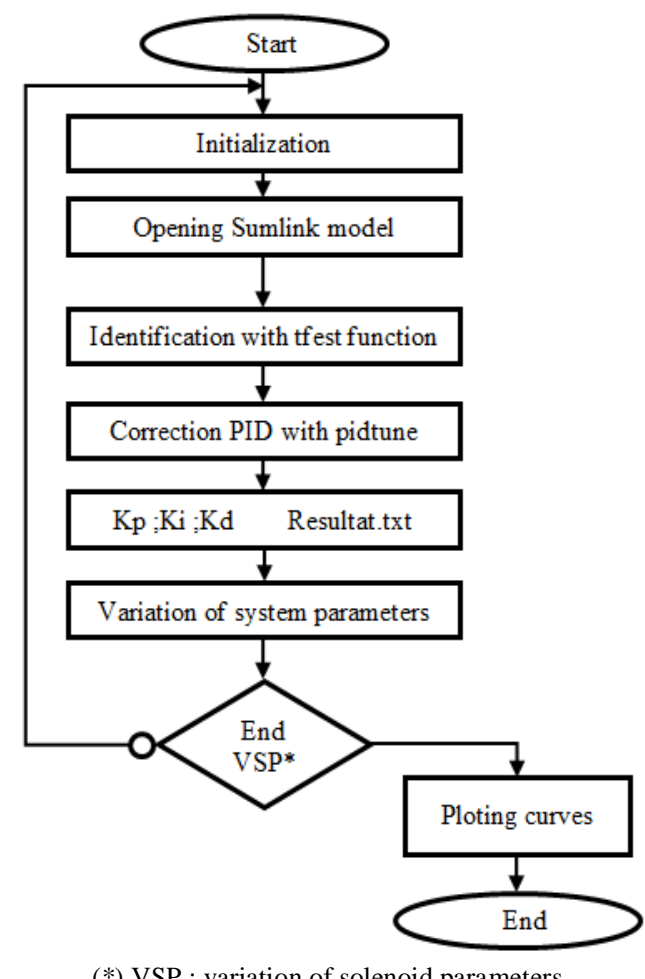

(*) VSP : variation of solenoid parameters

Figure 7. Simulation flowchart

\subsection{Simulation result for open loop system}

The Simulink model is simulated with the following parameters values: coil resistance $\mathrm{R}=100 \Omega$, spring stiffness constant $\mathrm{K}=2000 \mathrm{~N} / \mathrm{m}$, friction coefficient $\mathrm{B}=2 \mathrm{Ns} / \mathrm{m}$, mass plunger $\mathrm{M}=100 \mathrm{~g}$, wave length $\lambda=1550 \mathrm{~nm}$, fiber diameter $\mathrm{d}=125 \mu \mathrm{m}$ and the input light power $\mathrm{P} 0=1 \mathrm{~mW}$. Open loop step response of system is reported in the Figure 8 . This response shows that the system is stable but with damped oscillations which the overshot value is $79,6 \%$.

\subsection{Dynamic response in closed loop with corrector}

The close loop step response is represented in Figure 9. The PID controller parameters are obtained using pidtune function to correct the dynamic response with the constants values: $\mathrm{Kp}=2.8755, \mathrm{Ki}=757.5262$ and $\mathrm{Kd}=39193$. The controlled response is characterized by: overshot $=12,9 \%$, margin phase $=132$ and margin gain=inf.

\subsection{Influence of wave length variation on PID constants}

To evaluate the influence of the wavelength variation on the PID corrector parameters, we set the parameters of the solenoid $(\mathrm{m}=200 \mathrm{~g}, \mathrm{~K}=2000 \mathrm{~N} / \mathrm{m}, \mathrm{B}=2 \mathrm{Ns} / \mathrm{m}, \mathrm{L}=\mathrm{mH}, \mathrm{R}=10 \Omega$.) and varied the light source wavelength between $1100 \mathrm{~nm}$ and $2000 \mathrm{~nm}$. The parameters of the PID controller are determined to have a maximum output power, which corresponds to $\Delta \phi=2 \pi$ (6). The Figures 10-12 represent respectively the variation of $\mathrm{Kp}, \mathrm{Ki}$ and $\mathrm{Kd}$ as a function of the wavelength $\lambda$.

\subsection{Influence of solenoid parameters on PID constants}

We fix $\lambda=1550 \mathrm{~nm}$ and we vary the solenoid parameters ( $\mathrm{m}, \mathrm{K}$ and $\mathrm{B}$ ) to maintain a maximum output power with $\Delta \varphi=2 \pi$. We begin by first varying the electrical parameters ( $\mathrm{L}$ and $\mathrm{R}$ ) while keeping the mechanical parameters ( $\mathrm{m}, \mathrm{K}$ and $\mathrm{B}$ ) fixed, and then we do the opposite. The variation curves of the PID elements (Kp, Ki and $\mathrm{Kd}$ ) as a function of the solenoid parameters are given in the following Figures 13-24.

\subsection{Interpretation of results}

In case where solenoid parameters are fixed and those related to the fiber are changed (in this case the wavelength $\lambda$ ), the coefficients of the corrector PID are proportional to the wavelength with a coefficient of maximum proportionality $\mathrm{K}=500 \mathrm{~nm}-1$ for the constant integral $\mathrm{Ki}$. In case of varing the parameters of 
the solenoid level with $\lambda=1550 \mathrm{~nm}$ while maintaining $\mathrm{L}=20 \mathrm{mH}$ and $\mathrm{R}=10 \Omega$, we note from Figure 13 that the constant $\mathrm{Kp}$ is always low $[0,600]$ even if the stiffness and the coefficient of friction increases. For Kd, it is maximum in the Figure 14 for a value of the mass between 300 and $400 \mathrm{~g}$. As for the integration constant $\mathrm{Ki}$ and referring to Figures 15 and 16, we note that it remains constant and low $[0,300]$ for stiffness range 2:500 and mass range 50:100, even if the coefficient of friction augments.

For the coefficient of derivation $\mathrm{Kd}$, the graph of Figure 17 shows that it is inversely proportional to the coefficient of friction for a stiffness between 2 and 500. On the other hand, if the mass and friction increases the constant Kd decreases as shown in Figure 18. According to Figure 19, we note that the constant Kp keeps a low value ( 1 to 6 ) even if the mass and the stiffness increases. On the other hand, the constant Ki is proportional to the variations of the mass and the stiffness has a maximum value inferior to 900 as shown in Figure 20. Finally, for the variations of the electric quantities ( $\mathrm{L}$ and $\mathrm{R}$ ) while keeping the mechanical parameters $\mathrm{m}, \mathrm{k}$ and $\mathrm{B}$ constant, one note from the graphs of Figures 22, 23, 24 that the constants of the controller PID Ki, Kp and $\mathrm{Kd}$ are proportional to the value of the resistance $\mathrm{R}$ and inversely proportional to the value of inductance L. The results also show that the variation of the mechanical and electrical parameters affects the dynamic response of the stress on the fiber and therefore the intensity of the output power. Hence, it is necessary to provide an automatic adjustment of the PID gains in case of solenoid's parameters variations to keep response with sufficient stability.

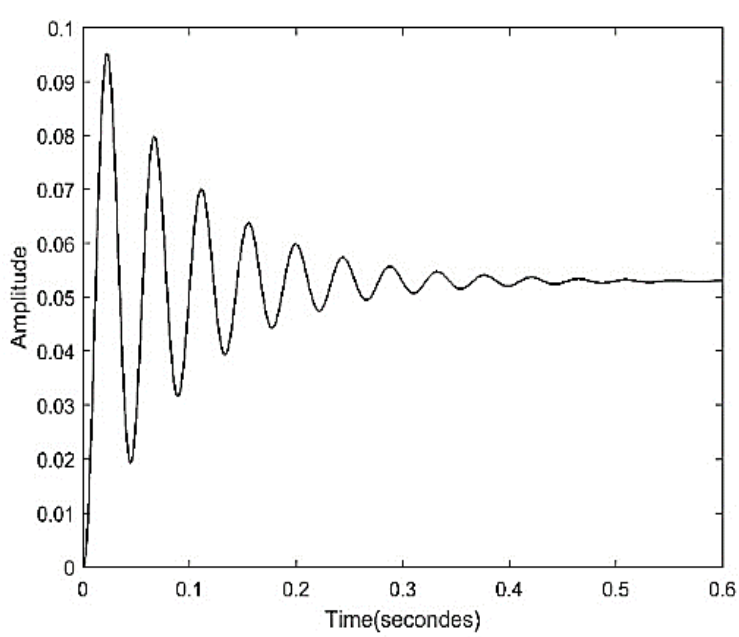

Figure 8. Open loop step response

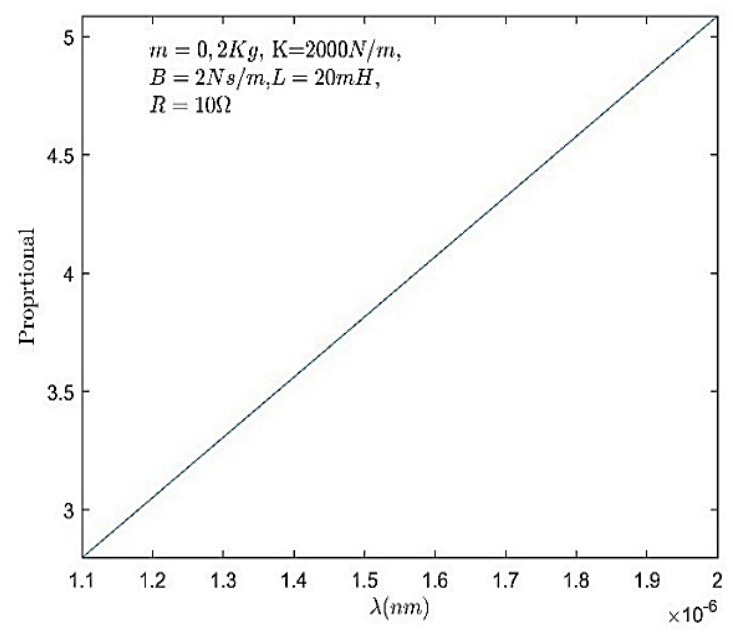

Figure 10. Variation of the gain $\mathrm{Kp}$ as a function of $\lambda$

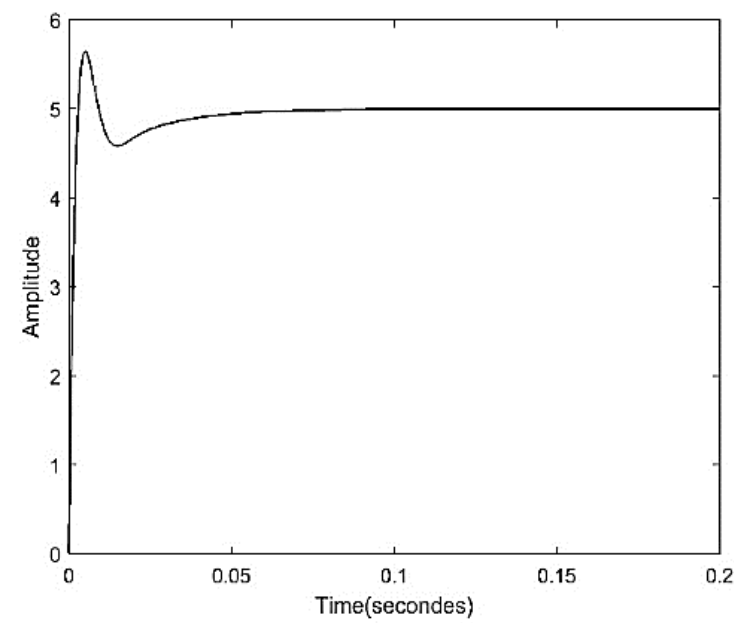

Figure 9. Dynamic response with PID controller

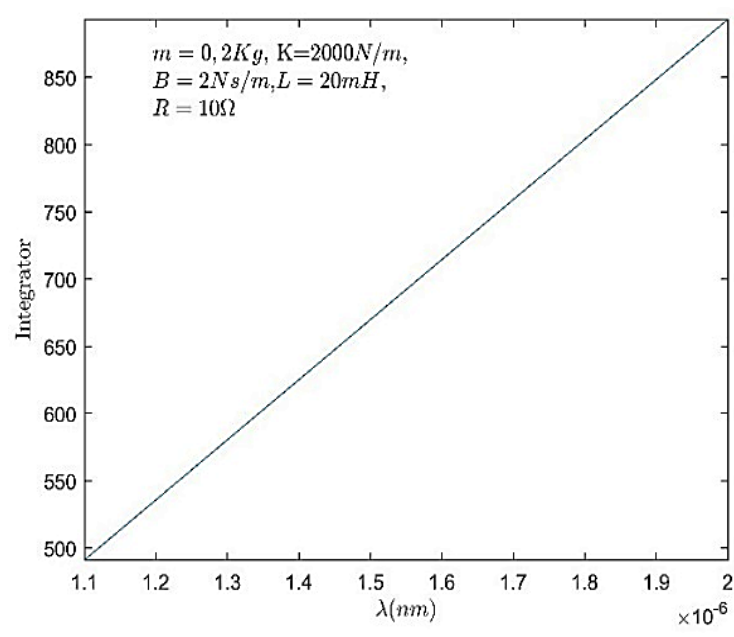

Figure 11. Variation of the gain $\mathrm{Ki}$ as a function of $\lambda$ 


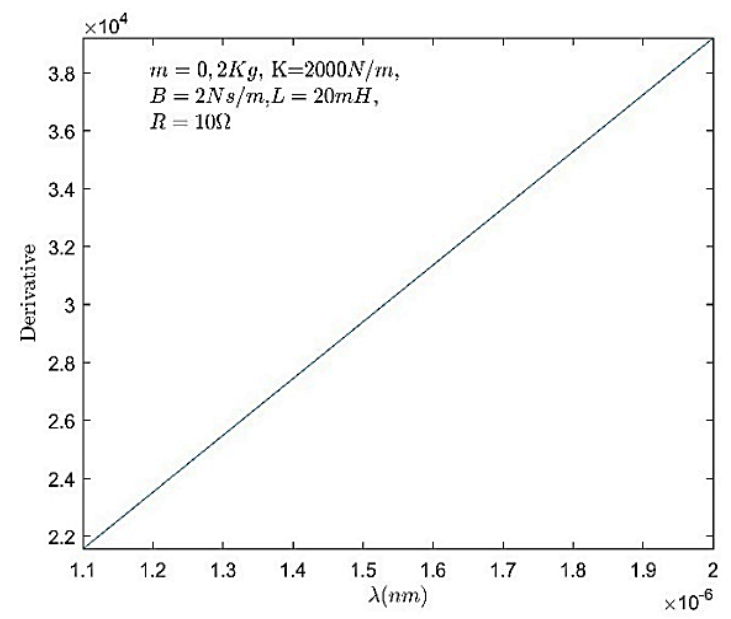

Figure 12. Variation of the gain $\mathrm{Kd}$ as a function of $\lambda$

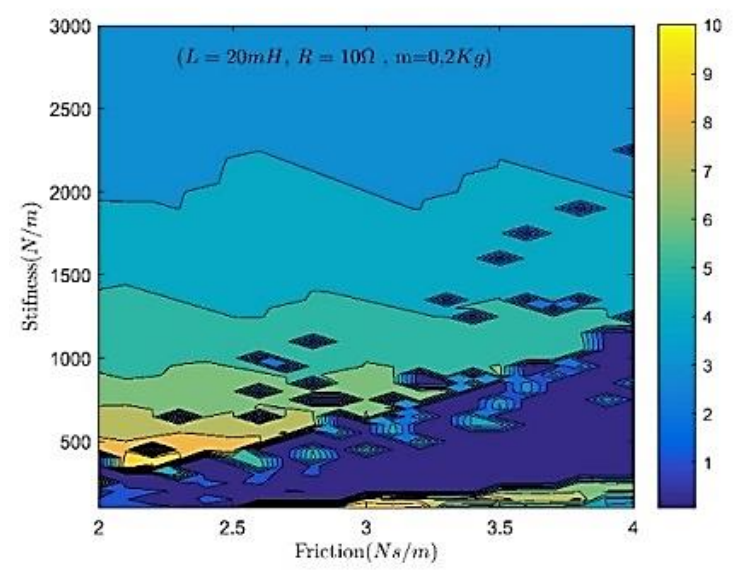

Figure 13. Map of Kp as function of stiffness and friction

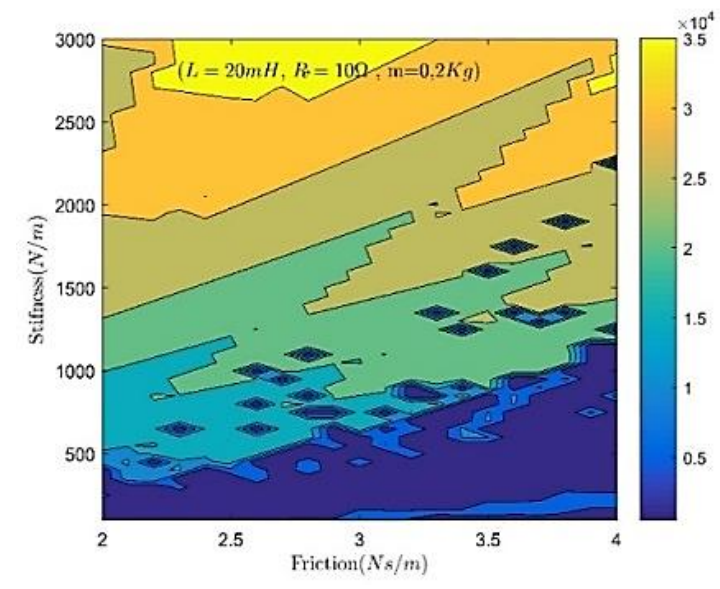

Figure 15. Map of Ki as function of stiffness and friction

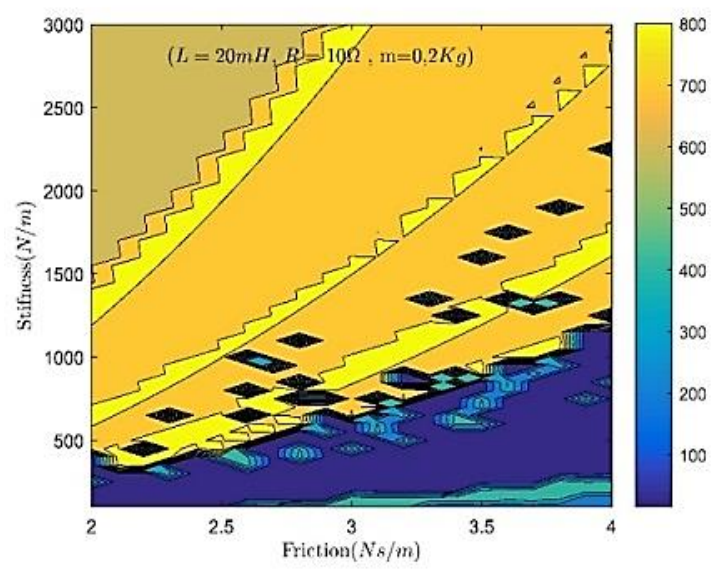

Figure 14. Map of Kd as function of stiffness and friction

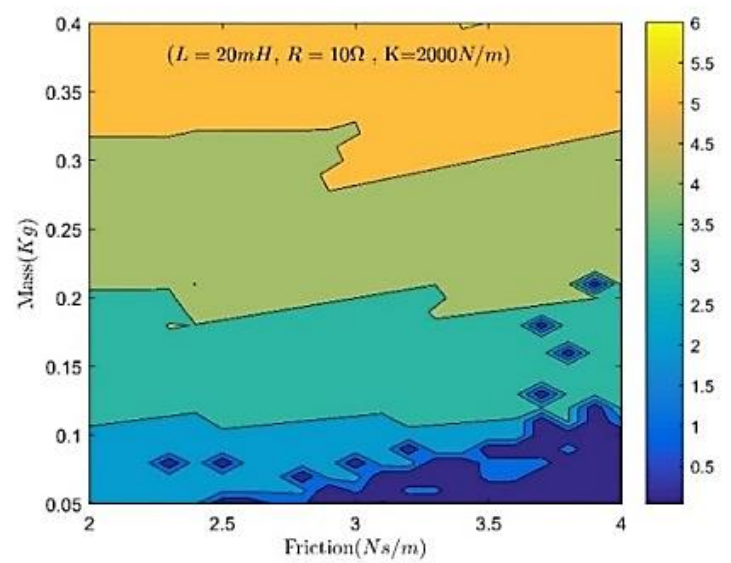

Figure 16. Map of $\mathrm{Ki}$ as function of masse and friction 


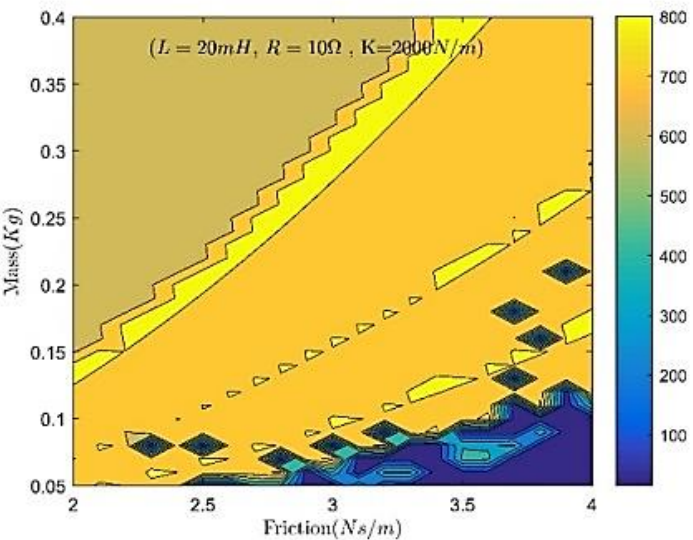

Figure 17. Map of $\mathrm{Kd}$ as function of masse and friction

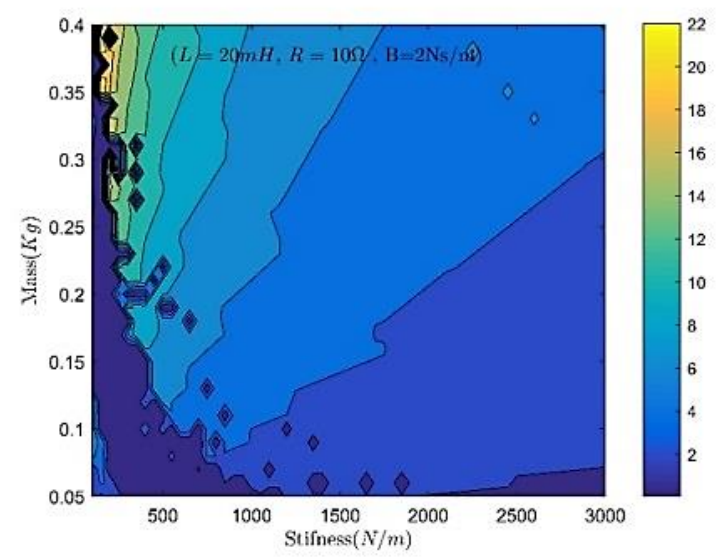

Figure 19. Map of Kp as function of masse and stifness

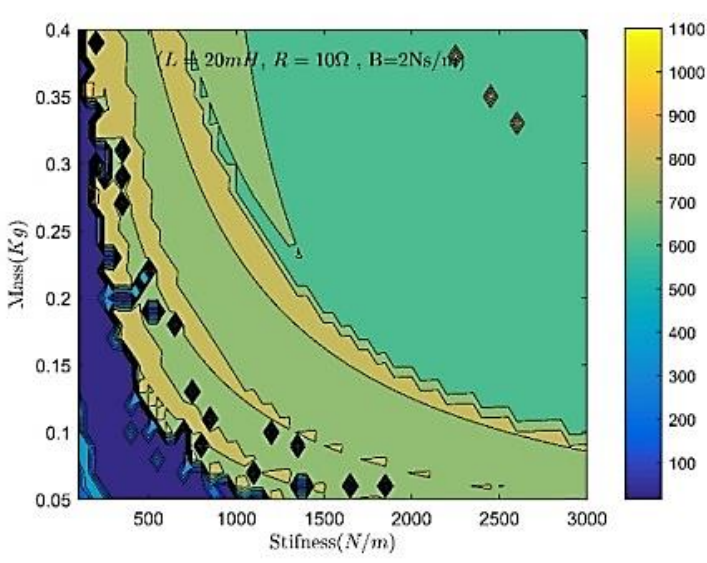

Figure 21. Map of Kd as function of masse and stifness

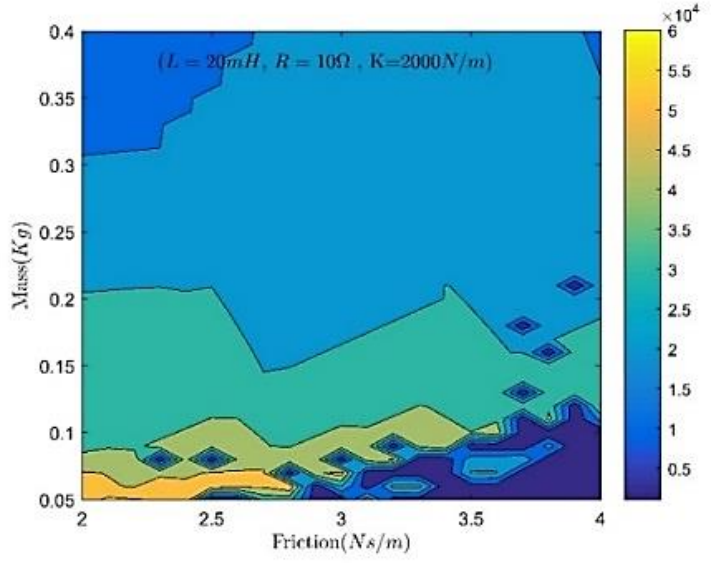

Figure 18. Map of $\mathrm{Kd}$ as function of masse and friction

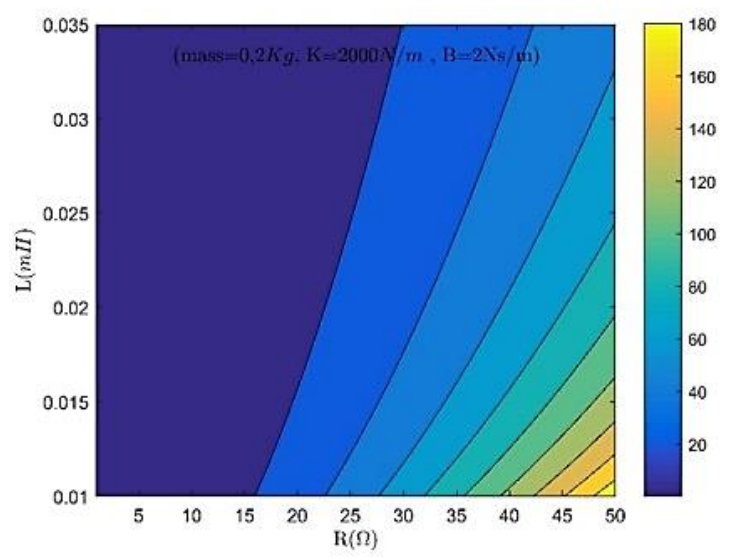

Figure 20. Map of $\mathrm{Ki}$ as function of masse and stiffness

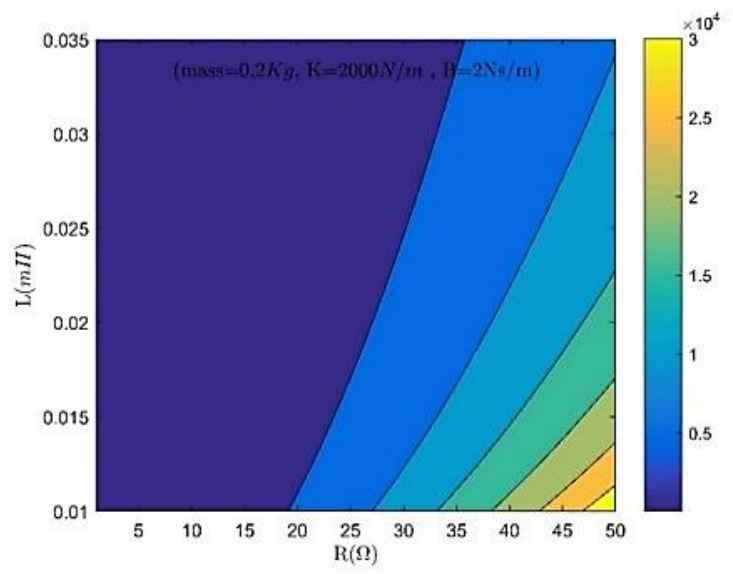

Figure 22. Map of Kp as function of $\mathrm{L}$ and $\mathrm{R}$ 


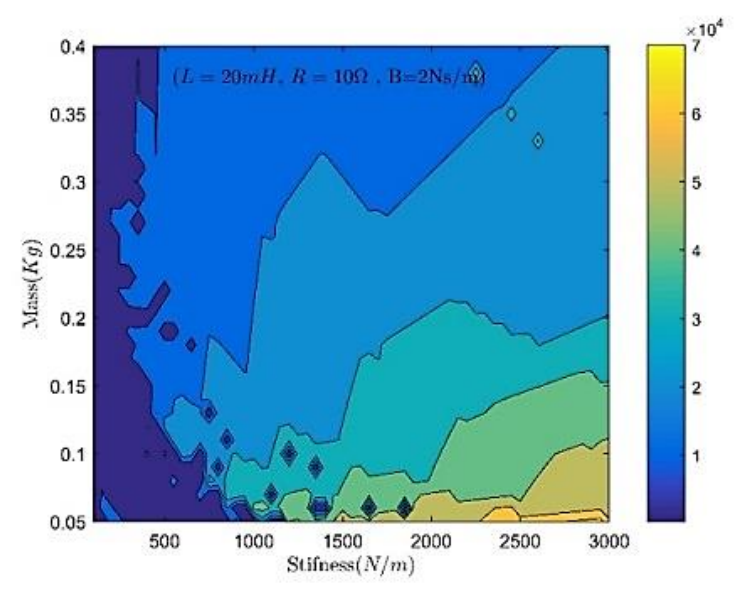

Figure 23. Map of Ki as function of $\mathrm{L}$ and $\mathrm{R}$

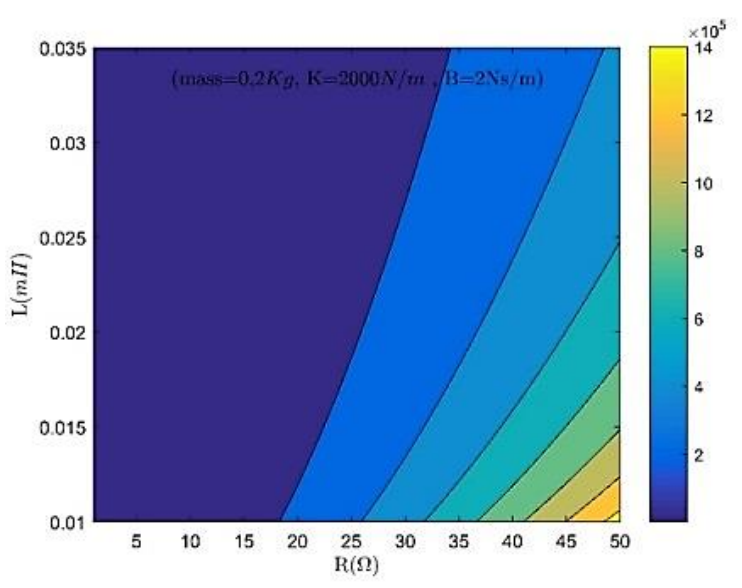

Figure 24. Map of $\mathrm{Kd}$ as function of $\mathrm{L}$ and $\mathrm{R}$

\section{CONCLUSION}

The electromagnetic fiber squeezer dynamic modeling analysis is presented with mathematical model to describe dynamic response. It is clear from this simulation study that the PID control theory provides a powerful control strategy in terms of transient response, indeed the results show that the over shoot is decreased greatly. We have also presented the influence of the system parameters and the control law for polarization controllers using solenoids as a fiber press. The results also show that mechanical and electrical system parameters variations as well as the wavelength affect the dynamic response of this type of polarization controllers. Automatic adjustment of the PID gains in case of solenoid parametric variations is realized by real time analyzis of the dynamic response with a PID corrector on a fiber by using a programmable system based on our program Matlab/Simulik. In case of parametric variation of the solenoid, this system will adjust PID gains and displays real time parameter values. This study based on the exploitation of solenoid in the optical field is very useful for monitoring in real time any variation of the parameters of this type of actuator used in others industrial or domestic system.

\section{REFERENCES}

[1] R. A. Steinberg, et al., "Performance limitations imposed on optical waveguide switches and modulators by polarization," Appl. Opt, vol. 15, no. 10, pp. 2440, Oct. 1976.

[2] R. Ulrich, "Polarization stabilization on single-mode fiber," Appl. Phys. Lett., vol. 35, no. 11, pp. 840-842, 1979.

[3] H. Heidrich, et al., "Polarisation transformer on Ti:LiNbO3 with reset-free optical operation for heterodyne/homodyne receivers," Electron. Lett., vol. 23, no. 7, pp. 335, 1987.

[4] T. Okoshi, et al., "Polarization-diversity receiver for heterodyne coherent optical fiber communications," in Proc. IOOC, paper 3OC3-2, pp. 386-387, 1983.

[5] T. G. Hodgkinson, et al., "polarisation insensitive heterodyne detection using polarisation scrambling," in Optical Fiber Communication, Reno, Nevada, pp. PDP15, 1987.

[6] L. J. Cimini, et al., "Polarisation-insensitive coherent lightwave system using wide-deviation FSK and data-induced polarisation switching," Electron. Lett., vol. 24, no. 6, pp. 358, 1988.

[7] Antao Chen, et al., "High performance lithium niobate polarization controller for PMD compensators," in The 15th Annual Meeting of the IEEE Lasers and Electro-Optics Society, Glasgow, UK, vol. 2, pp. 885-886, 2002.

[8] T. Imai, et al., "Optical polarisation control utilising an optical heterodyne detection scheme," Electron. Lett., vol. 21 , no. 2, pp. 52, 1985 .

[9] R. Noe, et al., "Endless polarization control systems for coherent optics," J. Light. Technol., vol. 6, no. 7, pp. 1199-1208, jul. 1988.

[10] M. Martinelli and R. A. Chipman, "Endless polarization control algorithm using adjustable linear retarders with fixed axes,” J. Light. Technol., vol. 21, no. 9, pp. 2089-2096, sep. 2003.

[11] S. H. Rumbaugh, et al., "Polarization control for coherent fiber-optic systems using nematic liquid crystals," J. Light. Technol., vol. 8, no. 3, pp. 459-465, mar. 1990.

[12] F. Heismann, "Integrated-optic polarization transformer for reset-free endless polarization control," IEEE J. Quantum Electron., vol. 25, no. 8, pp. 1898-1906, 1989.

[13] F. Mohr and U. Scholz, "Active polarization stabilization systems for use with coherent transmission systems or fibre-optic sensors," in Proc. 9th ECOC, pp. 313-316, 1983. 
[14] R. Ranganayakulu, G. U. B. Babu, and A. S. Rao, "Fractional filter IMC-PID controller design for an unstable inverted pendulum system," in 2017 IEEE International Conference on Smart Technologies and Management for Computing, Communication, Controls, Energy and Materials (ICSTM), Chennai, India, pp. 411-416, 2017.

[15] G.-Z. Zhao, et al., "Magneto-optic Crystal Polarization Controller Assisted Mode-Locked Fiber Laser," Chin. Phys. Lett., vol. 28, no. 3, pp. 034203, 2011.

[16] C. Li, H. Yang, et al., "Enhanced-Performance Control of an Electromagnetic Solenoid System Using a Digital Controller," IEEE Trans. Control Syst. Technol., vol. 24, no. 5, pp. 1805-1811, Sep. 2016.

[17] K. Ikeda, et al., "Endless Tracking Polarization Controller," Furukawa Review, no. 23, 2003.

[18] M.Y. Chen, et al., "A new design of asubmicropositioner utilizing electromagnetic actuators and flexure mech-anism," IEEE Trans. Ind. Electron., vol. 57, no. 1, pp. 96-106, Jan. 2010.

[19] T. Kajima and Y. Kawamura, "Development of a high-speed solenoid valve: Investigation of solenoids," IEEE Trans. Ind. Electron., vol. 42, no. 1, pp. 1-8, Feb. 1995.

[20] K. Mutschleret, et al., "Multi physics network simulation of a solenoid dispensing valve," Mechatronics, vol. 24, no. 3, pp. 209-221, 2014.

[21] L. Chu, et al., "Study onthe dynamic characteristics of pneumatic ABS solenoid valve for com-mercial vehicle," in Proc. IEEE Vehicle Power Propuls. Conf. (VPPC), pp. 641-644, Sep. 2007.

[22] A. Takai, et al., "Prototypingthe flexible solenoid-coil artificial muscle, for exoskeletal robots," in Proc. IEEE 13th Int. Conf. Control, Autom., Syst. (ICCAS), pp. 1046-1051, Oct. 2013

[23] K. W. Lim, et al, "Proportional control of a solenoid actuator," in Proc. IEEE 20th Int. Conf.Ind. Electron., Control, Instrum. (IECON), vol. 3, pp. 2045-2050.

[24] L. Yu and T. N. Chang, "Zero vibration on-off position control of dual solenoid actuator," IEEE Trans. Ind. Electron., vol. 57, no. 7, pp. 2519-2526, Jul. 2010.

[25] M. Taghizadeh, et al., "Modeling and identification of a solenoid valve for PW control applications," Compt. Rendus Mécanique, vol. 337, no. 3, pp. 131-140, 2009.

[26] T. Nguyen, et al., "Accurate sliding-mode control of pneumatic systems using low-cost solenoid valves," IEEE/ASME Trans. Mechatronics, vol. 12, no. 2, pp. 216-219, Apr. 2007.

[27] C. Nataraj and D. Lee, "Model-based adaptive control for a solenoid-valve system," in Proc. Int. Mech. Eng. Congr. Expo., pp. 1009-1015, 2010.

[28] Bureau International des Poids et Mesures et IEEE Instrumentation and Measurement Society, Éd., 2012 Conference on Precision Electromagnetic Measurements (CPEM 2012): Washington, DC, USA, 1-6 July 2012. Piscataway, NJ: IEEE, 2012.

[29] A. M. Smith, "Single-mode fibre pressure sensitivity," Electronics Letters, vol. 16, no. 20, pp. 773, 1980.

[30] D. Dong, X. Li, "Simulation and experimental research on the response of a novel high-pressure pneumatic pilotoperated solenoid valve," in Proc. IEEE 19th Int. Conf. Mechatron. Mach. Vis. Pract. (M2VIP), pp. 480-484, 2012. 